Bull. Austral. Math. Soc.

$53 \mathrm{~A} 05,53 \mathrm{A07}, 53 \mathrm{C} 40$

VOL. 42 (1990) [447-453]

\title{
RULED SURFACES OF FINITE TYPE
}

\section{Bang-Yen Chen, Franki Dillen, Leopold Verstraelen and Luc Vrancken}

Dedicated to Professor Tadashi Nagano on the occasion of his 60th birthday

We show that a ruled surface of finite type in a Euclidean space is a cylinder on a curve of finite type or a helicoid in Euclidean 3-space.

\section{INTRODUCTION}

A classical result of Catalan states that the only ruled minimal surfaces in Euclidean 3-space are the plane and the helicoid. It is well known that his result also holds for ruled minimal (2-dimensional) surfaces of any codimension in a Euclidean space, namely, the helicoid in $\mathbf{E}^{3}$ and the planes are the only minimal ruled surfaces in $\mathbf{E}^{n}$.

Minimal submanifolds of a Euclidean space are contained in a much larger class of submanifolds, namely in the class of submanifolds of finite type. Submanifolds of finite type are introduced by the first author in [1]. A submanifold $M^{n}$ of a Euclidean space $\mathbf{E}^{n+p}$ is said to be of finite type if each component of its position vector field $X$ can be written as a finite sum of eigenfunctions of the Laplacian $\Delta$ of $M^{n}$, that is, if

$$
X=X_{0}+\sum_{i=1}^{k} X_{i}
$$

where $X_{0}$ is a constant vector and $\Delta X_{i}=\lambda_{i} X_{i}$ for $i=1, \ldots, k$. If in particular all eigenvalues $\left\{\lambda_{1}, \lambda_{2}, \ldots, \lambda_{k}\right\}$ are mutually different, then $M^{n}$ is said to be of $k$-type. Note that every minimal submanifold of a Euclidean space is of 1-type, since $\Delta X=0$. If we define a polynomial $P$ by

$$
P(T)=\prod_{i=1}^{k}\left(T-\lambda_{i}\right)
$$

then $P(\Delta)\left(X-X_{0}\right)=0$.

\section{Received 12 December 1989}

The second and fourth authors are research assistants of the National Fund for Scientific Research (Belgium). The second author would like to thank The Michigan State University and the last two authors the University of Granada for their hospitality.

Copyright Clearance Centre, Inc. Serial-fee code: 0004-9729/90 \$A2.00+0.00. 
In this paper we consider ruled surfaces of finite type in a Euclidean space $\mathbf{E}^{n}$. From [9] we know that a 2-dimensional cone in $\mathbf{E}^{n}$ is of finite type if and only if it is a plane. Here we show that a ruled surface of finite type is either a cylinder on a curve of finite type in an affine hyperplane $\mathbf{E}^{n-1}$, or a minimal ruled surface, which therefore has to be a helicoid in $\mathbf{E}^{3}$. We shall prove the following theorem in Section 2.

TheOREM 1. A ruled surface $M$ in $\mathbf{E}^{n}$ is of finite type if and only if $M$ is a cylinder on a curve of finite type or $M$ is a part of a helicoid in an affine subspace $\mathbf{E}^{3}$.

If we restrict our attention to surfaces in Euclidean 3-space, we obtain that $M$ has to be a cylinder on a plane curve of finite type, or a helicoid. In Section 3 we shall show that a plane curve of finite type is a part of a circle or a line. Hence we obtain the following theorem.

ThEOREM 2. A ruled surface $M$ in $\mathbf{E}^{3}$ is of finite type if and only if $M$ is a part of a plane, a circular cylinder or a helicoid.

The following corollary is an immediate consequence of Theorem 2.

Corollary. A flat surface in $\mathbf{E}^{3}$ is of finite type if and only if it is a part of a plane or a circular cylinder.

In particular, every ruled surface of finite type in $\mathbf{E}^{3}$ is of 1-type or 2-type. This is not the case in $\mathbf{E}^{4}$. Indeed, in [6], the authors together with Deprez constructed examples of closed curves of $k$-type in $\mathbf{E}^{3}$ for any arbitrary $k$. A cylinder on such a curve is of $(k+1)$-type.

Up to this moment, the only known examples of surfaces of finite type in $\mathbf{E}^{3}$ are the plane, the sphere, the circular cylinder and the minimal surfaces. The first author proposed the following problem.

Problem. Classify all finite type surfaces in $\mathbf{E}^{3}$.

Concerning this problem, the first author has shown in [2] that a tube in $\mathbf{E}^{3}$ is of finite type if and only if it is a circular cylinder. In [4] the first and second authors showed that the only quadrics of finite type in $\mathbf{E}^{3}$ are the circular cylinders and the spheres. These results together with the results in this paper suggest that there are no other surfaces of finite type in $\mathbf{E}^{3}$, than the ones mentioned, but we hesitate to formulate this as a conjecture. On the other hand, the first author conjectured the following for compact surfaces.

CONJECTURE. The only compact finite type surfaces in $\mathbf{E}^{3}$ are the spheres.

Further support for this conjecture was obtained in [7]. At the end of this paper we make some short remarks concerning the rationality of curves of finite type. 


\section{RULED SURFACES OF FINITE TYPE}

Let $M$ be a ruled surface in $\mathbf{E}^{n}$. We consider two cases separately.

CASE 1. $M$ is a cylinder.

Suppose that $M$ is a cylinder over a curve $\gamma$ in an affine hyperplane $\mathbf{E}^{n-1}$, which we can choose to have the equation $x_{n}=0$. We can assume that $\gamma$ is parameterised by its arc length $s$. Then a parameterisation $X$ of $M$ is given by

$$
X(s, t)=\gamma(s)+t e_{n} .
$$

The Laplacian $\Delta$ of $M$ is given in terms of $s$ and $t$ by

$$
\Delta=-\frac{\partial^{2}}{\partial s^{2}}-\frac{\partial^{2}}{\partial t^{2}}
$$

and the Laplacian $\Delta^{\prime}$ of $\gamma$ is given by

$$
\Delta^{\prime}=-\frac{\partial^{2}}{\partial s^{2}}
$$

Certainly we have that $\Delta x_{n}=\Delta t=0$. Thus $M$ is of finite type if and only if each component of $\gamma(s)$ can be written as a finite sum of eigenfunctions of $\Delta$, that is,

$$
\gamma(s)=\Gamma_{0}+\sum_{i=1}^{k} \Gamma_{i}(s, t)
$$

where $\Delta \Gamma_{i}=\lambda_{i} \Gamma_{i}$. Assume that all the $\lambda_{i}$ are mutually different. If we apply $\prod_{i=2}^{k}\left(\Delta-\lambda_{i}\right)$ to $(1)$, we obtain that $\Gamma_{1}$ does not depend on $t$. Similarly we find that none of the $\Gamma_{i}$ depend on $t$. Moreover

$$
\Delta^{\prime} \Gamma_{i}(s)=-\frac{\partial^{2}}{\partial s^{2}} \Gamma_{i}(s)=-\frac{\partial^{2}}{\partial s^{2}} \Gamma_{i}(s)-\frac{\partial^{2}}{\partial t^{2}} \Gamma_{i}(s)=\Delta \Gamma_{i}(s)=\lambda_{i} \Gamma_{i}(s)
$$

for all $i$. Hence every component of $\gamma$ can be written as a finite sum of eigenfunctions of $\Delta^{\prime}$. This means that $\gamma$ is of finite type. Thus $M$ is of finite type if and only if $\gamma$ is of finite type. Moreover, if $\gamma$ is of $k$-type, then $M$ is of $(k+1)$-type, unless one of the eigenfunctions which appear in the decomposition of $\gamma$ has eigenvalue 0 , in which case $M$ is of $k$-type too.

CASE 2. $M$ is not cylindrical.

If $M$ is not cylindrical, we can decompose $M$ into open pieces such that on each piece we can find a parameterisation $X$ of the form

$$
X(s, t)=\alpha(s)+t \beta(s),
$$


where $\alpha$ and $\beta$ are curves in $\mathbf{E}^{n}$ such that $\left\langle\alpha^{\prime}, \beta\right\rangle=0,\langle\beta, \beta\rangle=1$ and $\left\langle\beta^{\prime}, \beta^{\prime}\right\rangle=1$. If we define a function $q$ by

$$
q=t^{2}+2\left\langle\alpha^{\prime}, \beta^{\prime}\right\rangle t+\left\langle\alpha^{\prime}, \alpha^{\prime}\right\rangle=\left\|\alpha^{\prime}+t \beta^{\prime}\right\|^{2},
$$

then it is easy to show that the Laplacian $\Delta$ of $M$ can be expressed as follows

$$
\Delta=-\frac{\partial^{2}}{\partial t^{2}}-\frac{1}{q} \frac{\partial^{2}}{\partial s^{2}}+\frac{1}{2} \frac{\partial q}{\partial s} \frac{1}{q^{2}} \frac{\partial}{\partial s}-\frac{1}{2} \frac{\partial q}{\partial t} \frac{1}{q} \frac{\partial}{\partial t} .
$$

The following lemma can be proved by a straightforward computation.

LEMMA. If $P$ is a polynomial in $t$ with functions in $s$ as coefficients and $\operatorname{deg}(P)=$ $d$, then

$$
\Delta\left(\frac{P(t)}{q^{m}}\right)=\frac{\widetilde{P}(t)}{q^{m+3}}
$$

where $\widetilde{P}$ is a polynomial in $t$ with functions in $s$ as coefficients and $\operatorname{deg}(\widetilde{P}) \leqslant d+4$.

From now on we suppose that $M$ is of $k$-type. Hence there exist numbers $c_{1}, \ldots, c_{k}$ such that

$$
\Delta^{k+1} X+c_{1} \Delta^{k} X+\cdots+c_{k} \Delta X=0 .
$$

We know that every component of $X$ is a linear function in $t$ with functions in $s$ as coefficients. By applying the Lemma, we easily obtain that

$$
\Delta^{r} X=\frac{P_{r}(t)}{q^{3 r-1}}
$$

where $P_{r}$ is a vector whose components are polynomials in $t$ with functions in $s$ as coefficients and $\operatorname{deg}\left(P_{r}\right) \leqslant 1+4 r$. Hence if $r$ goes up by one, the degree of the numerator of any component of $\Delta^{r} X$ goes up by at most 4 , while the degree of the denominator goes up by 6 . Hence the sum (2) can never be zero, unless of course $\Delta X=0$. But then $M$ is minimal. This proves Theorem 1 .

\section{Plane curves of finite type}

It is proved by the first author in [1] that every closed plane curve of finite type is of 1-type, and hence a circle. In [3], he states that every plane curve of finite type is a part of a circle or a line, without giving a proof. We give the proof here. Let $\gamma$ be a plane curve, parameterised by arc length $s$. Then the Laplacian $\Delta$ of $\gamma$ is given by $\Delta=-\partial^{2} / \partial s^{2}$. If $\gamma$ is of finite type, then $\gamma$ can be written as

$$
\gamma(s)=a_{0}+b_{0} s+\sum_{t=1}^{k_{1}}\left(a_{t} \cos \left(p_{t} s\right)+b_{t} \sin \left(p_{t} s\right)\right)+\sum_{t=1}^{k_{2}}\left(c_{t} e^{q_{t} s}+d_{t} e^{-q_{t} s}\right) .
$$


As indicated in [5] it follows from $\left\|\gamma^{\prime}\right\|=1$ that $c_{t}=d_{t}=0$. If $k_{1}=0$ then $\gamma$ is a straight line. If $k_{1}=1$ then $\gamma$ is a circle. So we assume from now on that $k_{1}>1$. We put $k=k_{1}$. We can suppose that $p_{1}<p_{2}<\cdots<p_{k}$ and that for every $t$, not both $a_{t}$ and $b_{t}$ are zero. Expressing the condition $\left\|\gamma^{\prime}\right\|=1$, we obtain similarly as in [1] that

$$
4 \sum_{\substack{i=1 \\ p_{i}=l}}^{k} p_{i} B_{i}-\sum_{\substack{i=1 \\ 2 p_{i}=l}}^{k} p_{i}^{2} A_{i i}-2 \sum_{\substack{i, j=1 \\ i>j \\ p_{i}+p_{j}=l}}^{k} p_{i} p_{j} A_{i j}+2 \sum_{\substack{i, j=1 \\ i=j \\ p_{i}-p_{j}=l}}^{k} p_{i} p_{j} D_{i j}=0
$$

and

$$
4 \sum_{\substack{i=1 \\ p_{i}=l}}^{k} p_{i} \bar{B}_{i}+\sum_{\substack{i=1 \\ 2 p_{i}=l}}^{k} p_{i}^{2} \bar{A}_{i i}+2 \sum_{\substack{i, j=1 \\ i>j \\ i}}^{k} p_{i} p_{j} \bar{A}_{i j}+2 \sum_{\substack{i, j=1 \\ i=1 \\ i=1}}^{k} p_{i} p_{j} \bar{D}_{i j}=0
$$

for all $l \in\left\{p_{i}+p_{j} \mid 1 \leqslant j \leqslant i \leqslant k\right\} \cup\left\{p_{i} \mid 1 \leqslant i \leqslant k\right\} \cup\left\{p_{i}-p_{j} \mid 1 \leqslant j<i \leqslant k\right\}$ where for all $i$ and $j, 1 \leqslant i, j \leqslant k$

$$
\begin{aligned}
B_{i} & =\left\langle b_{0}, b_{i}\right\rangle & \bar{B}_{i} & =\left\langle b_{0}, a_{i}\right\rangle \\
A_{i j} & =\left\langle a_{i}, a_{j}\right\rangle-\left\langle b_{i}, b_{j}\right\rangle & D_{i j} & =\left\langle a_{i}, a_{j}\right\rangle+\left\langle b_{i}, b_{j}\right\rangle \\
\bar{A}_{i j} & =\left\langle a_{i}, b_{j}\right\rangle+\left\langle a_{j}, b_{i}\right\rangle & \bar{D}_{i j} & =\left\langle a_{i}, b_{j}\right\rangle-\left\langle a_{j}, b_{i}\right\rangle .
\end{aligned}
$$

For $l=2 k$ we obtain from $(\mathrm{I}(l))$ and $(\mathrm{J}(l))$ that $A_{k k}=\bar{A}_{k k}=0$. Thus $a_{k}$ and $b_{k}$ are orthogonal and have the same length. Hence we can choose coordinates on $\mathbf{E}^{2}$ such that $a_{k}=(u, 0)$ and $b_{k}=(0, u)$. Similarly from $\left(\mathrm{I}\left(p_{k}+p_{k-1}\right)\right)$ and $\left(\mathrm{J}\left(p_{k}+p_{k-1}\right)\right)$, we obtain that $A_{k, k-1}=\bar{A}_{k, k-1}=0$. This shows that $a_{k-1}=\left(u_{k-1}, v_{k-1}\right)$ and $b_{k-1}=\left(-v_{k-1}, u_{k-1}\right)$ for some $u_{k-1}$ and $v_{k-1}$.

Now assume $a_{t}=\left(u_{t}, v_{t}\right)$ and $b_{t}=\left(-v_{t}, u_{t}\right)$ for all $t>r$. We show that $a_{r}$ and $b_{r}$ are also of this form. Indeed, by the assumption, we have that $A_{i j}=\bar{A}_{i j}=0$ for all $i, j>r$. But then it follows from $\left(\mathrm{I}\left(p_{k}+p_{r}\right)\right)$ and $\left(\mathrm{J}\left(p_{k}+p_{r}\right)\right)$ that $A_{k, r}=$ $\bar{A}_{k, r}=0$. This implies the assertion. Hence $a_{t}=\left(u_{t}, v_{t}\right)$ and $b_{t}=\left(-v_{t}, u_{t}\right)$ for all $t \in\{1, \ldots, k\}$.

Next, we obtain from $\left(\mathrm{I}\left(p_{k}\right)\right)$ and $\left(\mathrm{J}\left(p_{k}\right)\right)$ that $B_{k}=\bar{B}_{k}=0$, so that $b_{0}=0$. Finally we obtain from $\left(\mathrm{I}\left(p_{k}-p_{1}\right)\right)$ and $\left(\mathrm{J}\left(p_{k}-p_{1}\right)\right)$ that $D_{k 1}=\bar{D}_{k 1}=0$, so that $a_{1}=b_{1}=0$. This is a contradiction.

Hence the following theorem is proved, which was also obtained in [8].

THEOREM 3. Let $\gamma$ be a plane curve of finite type. Then $\gamma$ is a part of a circle or a part of a straight line.

To finish this paper we show that every closed curve of finite type is rational. 
THEOREM 4. Let $\gamma$ be a closed curve of finite type. Then $\gamma$ is a rational curve.

Proof: A closed curve of finite type can be written as

$$
\gamma(s)=a_{0}+\sum_{t=1}^{k}\left(a_{t} \cos \left(p_{t} \frac{s}{r}\right)+b_{t} \sin \left(p_{t} \frac{s}{r}\right)\right)
$$

whereby $p_{1}, p_{2}, \ldots, p_{k}$ are positive integers. Let $d=\operatorname{gcd}\left(p_{1}, p_{2}, \ldots, p_{k}\right)$. Using Legendre polynomials, we immediately find that (the components of) $\gamma(s)$ can be written as a polynomial of degree $p_{t} / d$ in $\cos (d s / r)$ and $\sin (d s / r)$. If we make the substitutions

$$
\cos \left(d \frac{s}{r}\right)=\frac{1-t^{2}}{1+t^{2}}, \quad \sin \left(d \frac{s}{r}\right)=\frac{2 t}{1+t^{2}}
$$

it follows that $\gamma$ is a rational curve of degree $2 p_{k} / d$.

The number $p_{k}$ is sometimes called the upper order of $\gamma$. So a closed curve of finite type is a rational curve of degree twice the upper order, at least if the curve is not run through more than once.

Theorem 4 is not valid if the curve is not closed. Indeed, a helix in $\mathbf{E}^{3}$ is of 2-type, but it is not rational.

\section{REFERENCES}

[1] B.-Y. Chen, Total mean curvature and submanifolds of finite type (World Scientific, Singapore, 1984).

[2] B.-Y. Chen, 'Surfaces of finite type in Euclidean 3-space', Bull. Soc. Math. Belg. Sér. B 39 (1987), 243-254.

[3] B.-Y. Chen, 'Null 2-type surfaces in Euclidean space', in Algebra, Analysis and Geometry, Proc. of Symp. in honor of C.J. Hsu and K.S. Shih, pp. 1-18 (World Scientific, Singapore, 1988).

[4] B.-Y. Chen and F. Dillen, 'Quadrics of finite type', J. Geom. 38 (1990), 16-22.

[5] B.-Y. Chen and F. Dillen, 'Surfaces of finite type and constant curvature in the 3-sphere', C.R. Math. Rep. Acad. Sci. Canad 12 (1990), 47-49.

[6] B.-Y. Chen, J. Deprez, F. Dillen, L. Verstraelen and L. Vrancken, 'Curves of finite type', in Geometry and Topology of Submanifolds II, pp. 76-110, 1990.

[7] F. Dillen, J. Pas and L. Verstraelen, 'On surfaces of finite type in Euclidean 3-space', Kodai Math. J. 13 (1990), 10-21.

[8] A. Ferrández and P. Lucas, (personal communication).

[9] O. Garay, 'Finite type cones shaped on spherical submanifolds', Proc. Amer. Math. Soc. 104 (1988), 868-870. 
Michigan State University Department of Mathematics Well's Hall

East Lansing, MI 48824

United States of America
Katholieke Universiteit Leuven Departement Wiskunde

Celestijnenlaan $200 \mathrm{~B}$

B-3030 Leuven

Belgium 